\title{
Schrödinger-equation formalism for a dissipative quantum system
}

\author{
E. Anisimovas* $*$ \\ Department of Theoretical Physics, Vilnius University, Sauletekio al. 9, LT-10222 Vilnius, Lithuania \\ A. Matulis $\oplus$ \\ Semiconductor Physics Institute, Goštauto 11, LT-01108 Vilnius, Lithuania
}

(Dated: October 12, 2018)

\begin{abstract}
We consider a model dissipative quantum-mechanical system realized by coupling a quantum oscillator to a semi-infinite classical string which serves as a means of energy transfer from the oscillator to the infinity and thus plays the role of a dissipative element. The coupling between the two - quantum and classical — parts of the compound system is treated in the spirit of the meanfield approximation and justification of the validity of such an approach is given. The equations of motion of the classical subsystem are solved explicitly and an effective dissipative Schrödinger equation for the quantum subsystem is obtained. The proposed formalism is illustrated by its application to two basic problems: the decay of the quasi-stationary state and the calculation of the nonlinear resonance line shape.

PACS numbers: 03.65.Sq, 03.65.Yz, 73.21.La
\end{abstract}

\section{INTRODUCTION}

The rapid expansion of research frontiers into the nanometer and femtosecond regime demands a careful consideration of dissipation as a way to counterbalance the energy influx from the external fields in quantum mechanical systems.

The subject takes roots in the seminal work by Feynman and Vernon [1] who treated a quantum object coupled to an infinite collection of oscillators as a model of a linear dissipative environment. By employing the path-integral techniques they were able to eliminate the environment variables and arrived at a dissipative modification of the Green's function of the quantum object. This approach was further used by a number of authors, most notably by Caldeira and Leggett [2], who applied it to a specific problem of dissipative effects in tunneling on a macroscopic scale. A detailed account on the problem of dissipation in quantum-mechanical systems can be found in a monograph by Weiss [3] while semiclassical approaches are also reviewed in [4].

Generally, the main mode of attack uses the densitymatrix formalism [5] and the popular Lindblad technique [6]. This approach is relevant for open quantum systems. In contrast, the considerably less arduous Schrödingerequation formalism is regarded to be appropriate for isolated systems [7] that are described by a hermitian Hamiltonian.

Despite this fact, there were successful attempts to include dissipation directly in the Schrödinger equation. Most often they based on the de Broglie-Bohm picture [8] and employed modifications to the quantum potential [9]. These ideas were used by Kostin to derive a

\footnotetext{
*Electronic address: egidijus.anisimovas@ff.vu.lt
}

†Electronic address: amatulis@takas.lt dissipative nonlinear Schrödinger equation [10], and Albrecht [11] worked out the general principles limiting the acceptable form of the nonlinear terms.

The problem of dissipation in quantum mechanics is closely connected to the use of mixed quantum-classical treatments [12, 13] whereby only a few degrees of freedom of a complex system are treated quantum-mechanically while the remaining ones are described classically. Although Egorov and coworkers [14] point out that not always an accurate description is attained and so this approach must be used with care, it is recognized as a method of choice when a significant reduction of complexity is desired.

In this paper we present a derivation of a rather simple nonlinear Schrödinger equation that includes a dissipative term. This equation is obtained from a consideration of a physical model: a quantum oscillator connected to an infinite classical string that acts as a means to transfer energy away from the quantum system (see also [15]). The obtained Schrödinger-like equation is illustrated by considering two specific examples. The first one, the decay of a quasi-stationary state, is an auxiliary one and shows that the results obtained by using our analysis do not contradict the ones derived by the usual and more complex methods using the density-matrix formalism and a double set of variables. The second example, the nonlinear resonance, is interesting on its own and demonstrates that the quantum-mechanical nonlinear resonance is physically richer than its classical analog.

Our paper is organized in the following way. In Sect. II, the model system is introduced and the dissipative Schrödinger-like equation for the quantum system is derived. In Sect. III, the obtained equation is simplified employing the rotating-wave approximation. In the next two Sections, some illustrations of the developed technique are presented: in Sect. IV we consider the decay of the quasi-stationary state, and Sect. $\mathrm{V}$ is devoted to the non-linear resonance. Finally, our conclusions are 
formulated in Sect. VI.

\section{INTERACTION OF THE QUANTUM OSCILLATOR WITH A CLASSICAL CHAIN}

Let us begin by introducing our model of weakly coupled quantum and classical subsystems. The quantum system is modeled as a harmonic oscillator of mass $m$ and spring constant $k$, described by the quantum-mechanical position and momentum operators $\hat{x}$ and $\hat{p}$. The role of the classical system is played by a semi-infinite classical string which, for the sake of convenience, is discretized and represented as a chain of identical balls of mass $M$ interconnected by springs of equilibrium length $a$ and spring constant $K$. The two subsystems are linked by a different spring (spring constant $\kappa$ ) connecting the quantum oscillator to the ball sitting at the end of the chain as depicted in Fig. 1. The corresponding Hamiltonian reads

$$
\begin{aligned}
H= & \frac{1}{2 m} \hat{p}^{2}+\frac{k}{2} \hat{x}^{2}+\frac{\kappa}{2}\left(\hat{x}-x_{0}\right)^{2} \\
& +\sum_{n=0}^{\infty}\left[\frac{1}{2 M} p_{n}^{2}+\frac{K}{2}\left(x_{n}-x_{n+1}\right)^{2}\right],
\end{aligned}
$$

with $x_{n}$ denoting the coordinate of the $n$-th ball measured from its equilibrium position, and $p_{n}$ the respective momentum.

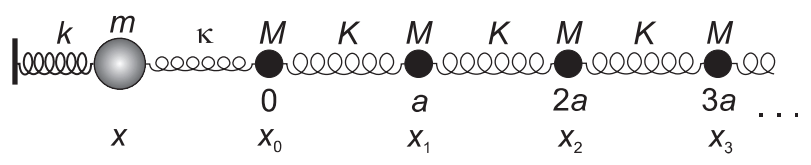

FIG. 1: The layout of the model system.

Following [16], we assume that the quantum oscillator is described by its own wave function $\Psi(x, t)$ which solves the Schrödinger equation

$$
\beta \hbar \frac{\partial}{\partial t} \Psi(x, t)=H \Psi(x, t)
$$

with the above Hamiltonian (1) while the dynamical variables of the balls in the chain obey the classical Hamilton equations

$$
\dot{x}_{n}=\frac{\partial}{\partial p_{n}}\langle H\rangle, \quad \dot{p}_{n}=-\frac{\partial}{\partial x_{n}}\langle H\rangle,
$$

with the Hamiltonian operator replaced by its quantummechanical average over the state of the quantum oscillator

$$
\langle H\rangle=\int_{-\infty}^{\infty} d x \Psi^{*}(x, t) H \Psi(x, t) .
$$

In this way, quantum variables and operators do not enter the equations for the classical degrees of freedom and one obtains a consistent description. This averaging constitutes the main assumption of the quasiclassical approximation and was used earlier to treat the coupling of classical and quantum degrees of freedom [16]. We give some further justification of this crucial step below.

Eqs. (3) lead to the standard classical equations for the chain

$$
\begin{aligned}
M \ddot{x}_{0}+K\left(x_{0}-x_{1}\right)+\kappa x_{0} & =\kappa\langle x\rangle, \\
M \ddot{x}_{n}-K\left(x_{n-1}-2 x_{n}+x_{n+1}\right) & =0, n \geqslant 1 .
\end{aligned}
$$

The second of these equations (5b) is readily solved by the Fourier transform

$$
x_{n}=\frac{1}{2 \pi} \int_{-\infty}^{\infty} d q \mathrm{e}^{\beta(q a n-\omega t)} f(q),
$$

producing the standard dispersion law

$$
\omega^{2}=\frac{4 K}{M} \sin ^{2}(q a / 2) .
$$

From here on we restrict our consideration to the longwavelength approximation $(q a \ll 1)$ thus neglecting the discretization and effectively returning to a continuousstring model for the classical subsystem. In this limit, we have a linear dispersion law

$$
\omega=v q
$$

with the wave-propagation velocity $v=\Omega a$ and $\Omega=$ $\sqrt{K / M}$.

Inserting the Fourier transform (6) into the boundary condition (5a) and using the long-wavelength approximation we are able to simplify its left-hand side to

$$
\begin{aligned}
& \frac{1}{2 \pi} \int_{-\infty}^{\infty} d q \mathrm{e}^{-\beta \omega t}\left\{K\left(1-\mathrm{e}^{\beta q a}\right)-M v^{2} q^{2}+\kappa\right\} f(q) \\
& \approx \frac{1}{2 \pi} \int_{-\infty}^{\infty} d q \mathrm{e}^{\beta v q t}(-i K q a+\kappa) f(q) \\
& =\frac{K a}{v} \dot{x}+\kappa x_{0} .
\end{aligned}
$$

The resulting equation of motion for the first ball coordinate $x_{0}$

$$
\frac{K a}{v} \dot{x}_{0}+\kappa x_{0}=\kappa\langle x\rangle
$$

may be interpreted as the equation of motion for a dissipative classical mode. Eq. (10) together with the Schrödinger equation (2) for the quantum variables constitute the complete equation set describing the behavior of the dissipative quantum system.

Now we turn to the examination of the validity of the employed quasiclassical approximation. A smooth way to proceed is to introduce the dimensionless variables by means of scaling

$$
\begin{aligned}
\hat{x} \rightarrow l \hat{x}, \quad \hat{p} \rightarrow \frac{\hbar}{l} \hat{p}, \quad x_{0} \rightarrow l_{0} x_{0}, \quad t \rightarrow \omega_{0}^{-1} t, \\
\omega_{0}=\sqrt{\frac{k}{m}}, \quad l=\sqrt{\frac{\hbar}{m \omega_{0}}}, \quad l_{0}=\sqrt{\frac{\hbar}{M \Omega}} .
\end{aligned}
$$


Here $l$ and $l_{0}$ are, respectively, the characteristic length scales of the quantum and classical subsystems, and $\omega_{0}$ stands for the frequency of the quantum oscillator. The scaling enables us to rewrite the obtained set of equations as

$$
\begin{aligned}
& \text { В } \frac{\partial}{\partial t} \Psi(x, t)=H \Psi(x, t), \\
& H=\frac{1}{2} \hat{p}^{2}+\frac{1}{2} \hat{x}^{2}-\lambda \hat{x} x_{0}, \\
& \dot{x}_{0}+\lambda \xi x_{0}=\lambda\langle x\rangle,
\end{aligned}
$$

with

$$
\lambda=\frac{\kappa}{k_{0}} \sqrt{\frac{\omega_{0} m}{\Omega M}}, \quad \xi=\frac{l_{0}}{l} .
$$

Note that we excluded the terms which do not depend on the operators $\hat{x}$ and $\hat{p}$ from the quantum Hamiltonian (12b) as they can always be absorbed into the wavefunction phase. The obtained equations describe the interaction of a quantum-mechanical system with a single dissipative classical mode $x_{0}$. In fact, the derivation does not depend on the assumption that the quantum system is a harmonic oscillator. Thus, the equations are general and can be applied to any quantum system with dissipation. For instance, considering an anharmonic oscillator driven by an external time-periodic force (we treat this case as an illustration) the above Hamiltonian is supplemented with the following additional terms

$$
\Delta H=-\hat{x} f \cos (\omega t)+\alpha \hat{x}^{4},
$$

with $f$ expressing the force amplitude and $\alpha$ being the anharmonicity coefficient.

In order to have an appreciable (that is, not negligibly small) interaction between the quantum and classical subsystems one has to assume the characteristic frequencies $\omega_{0}$ and $\Omega$ to be of the same order of magnitude. Under this assumption the quantum oscillator will be able to emit "phonons" into the string. Furthermore, the coupling constant $\kappa$ representing the interaction of quantum oscillator with the chain should not exceed the constant $k$ describing the oscillator potential itself. Otherwise, the oscillator cannot be considered as a separate entity. In view of the above constraints, the dimensionless coupling constants $\lambda$ and $\xi$ depend essentially only on the adiabatic parameter $m / M$.

Thus, in the case of a light quantum oscillator interacting with a chain of heavy balls the above adiabatic parameter is small and so are the coupling constants. The smallness of the coupling constant ensures a weak perturbation of the chain. The chain under consideration may be treated as a collection of noninteracting harmonic modes. Meanwhile, the quantum-mechanical description of a harmonic mode gives the same result as the classical one. Therefore, one may conclude that the smallness of the adiabatic parameter $m / M$ guarantees the validity of the applied quasiclassical approximation for the description of the interaction between the quantum and classical subsystems. A similar argument was also presented in the development of the mixed quantumclassical dynamics [12].

Alternatively, one may argue that due the smallness of the adiabatic parameter the characteristic length of the wave functions of heavy balls $l_{0}$ is much smaller than the characteristic length $l$ of the wave function of the oscillator. Consequently, the balls may be considered to be following their well-defined classical trajectories whereas the oscillator has to be described quantum-mechanically.

\section{ROTATING-WAVE APPROXIMATION}

Let us now proceed to some illustrations of the developed formalism based on a weakly nonlinear oscillator. Namely, we assume that the Hamiltonian of the quantum subsystem consists of a sum of Eq. (12b) and Eq. (14). Besides, we restrict the treatment to the case of a weak nonlinearity, that is, we assume that the anharmonicity $\alpha$, the force amplitude $f$, and the coupling constants $\lambda$ and $\xi$ are small quantities compared to the unity. We also restrict the frequency of the driving field to the immediate vicinity of the resonant frequency of the harmonic oscillator which is unity in the dimensionless units. Thus we are able to treat the frequency deviation from the resonance as yet another small parameter $\eta=\omega-1$.

We expand the sought wave function into the series of harmonic-oscillator eigenfunctions

$$
\Psi(x, t)=\sum_{n=0}^{\infty} a_{n}(t) \mathrm{e}^{-\beta \omega E_{n} t} \psi_{n}(x) .
$$

Here, the dimensionless energies and the corresponding eigenfunctions read

$$
E_{n}=n+\frac{1}{2}, \quad \psi_{n}(x)=\frac{1}{\sqrt{2^{n} n ! \sqrt{\pi}}} \mathrm{e}^{-x^{2} / 2} H_{n}(x),
$$

and the symbol $H_{n}(x)$ stands for the $n$-th Hermite polynomial. Note that instead of the usual time dependence $\sim \mathrm{e}^{-\beta E_{n} t}$ of a harmonic-oscillator eigenfunction Eq. (15) features a slightly modified exponential factor $\mathrm{e}^{-\beta \omega E_{n} t}$ that absorbs a part of the time dependence of the expansion coefficient $a_{n}(t)$.

Using the above expansion of the wave function (15) the coordinate average can be presented as

$$
\begin{aligned}
\langle x\rangle & =\sum_{n, m=0}^{\infty} \mathrm{e}^{\beta \omega\left(E_{n}-E_{m}\right) t} a_{n}^{*} a_{m}\langle n|x| m\rangle \\
& =\sqrt{2} \operatorname{Re}\left(\mathrm{e}^{\beta \omega t} a\right)
\end{aligned}
$$

where

$$
a=\sum_{n=0}^{\infty} a_{n} a_{n+1}^{*} \sqrt{n+1}
$$


is the usual polarization of the quantum oscillator.

Inserting the coordinate average (17) into Eq. (12c), and solving it to the leading term in the small coupling constants we obtain the following expression for the coordinate of the first ball

$$
x_{0}=\sqrt{2} \lambda \int d t \operatorname{Re}\left(\mathrm{e}^{\beta \omega t} a\right)=\sqrt{2} \lambda \operatorname{Im}\left(\mathrm{e}^{\beta \omega t} a\right) .
$$

Next, inserting the obtained expression (19) and the wave function (15) into Eq. (12a), projecting this equation onto the $n$-th state, and neglecting all oscillating terms (this amounts to the usual rotating-wave approximation) we arrive at the final set of equations for the time evolution of the wave function expansion coefficients

$$
\begin{aligned}
& ß \dot{a}_{n}=-\left\{\eta E_{n}-\alpha\left\langle n\left|x^{4}\right| n\right\rangle\right\} a_{n} \\
& -\sqrt{n}\left(F+ß \gamma a^{*}\right) a_{n-1}-\sqrt{n+1}(F-ß \gamma a) a_{n+1} .
\end{aligned}
$$

Here, for the sake of simpler notation we denoted

$$
F=f / 2 \sqrt{2}, \quad \gamma=\lambda^{2} / 2 .
$$

The obtained set of equations (20) was solved in the two- and three-level approximations, and the results are presented in the following sections.

\section{DECAY OF THE QUASI-STATIONARY STATE}

In order to illustrate the validity of the proposed method we consider a well-known problem of the decay of a quasi-stationary state.

For this purpose, we assume that the quantum system has only two states and there is no anharmonicity and no external force $(F=\eta=\alpha=0)$. At the beginning the system is prepared in the upper level, that is, the boundary condition reads

$$
t=-\infty, \quad a_{0}=0, \quad a_{1}=1 .
$$

According to Eq. (20), the behavior of this simple system is described by the following two equations

$$
\dot{a}_{0}=\gamma a a_{1}, \quad \dot{a}_{1}=-\gamma a^{*} a_{0}, \quad \text { with } a=a_{0} a_{1}^{*} .
$$

These equations are readily solved analytically, and the solution reads

$$
\left|a_{0}\right|^{2}=\frac{1}{\mathrm{e}^{-2 \gamma t}+1}, \quad\left|a_{1}\right|^{2}=\frac{1}{\mathrm{e}^{2 \gamma t}+1} .
$$

These filling factors are shown in Fig. 2 by the thick solid (excited level) and dashed (ground level) lines. We see that the quantum transition takes place during a finite period of time proportional to $\gamma^{-1}$, the reciprocal friction coefficient. The dotted curve shows the oscillator polarization

$$
a=\frac{1}{2 \cosh (\gamma t)}
$$

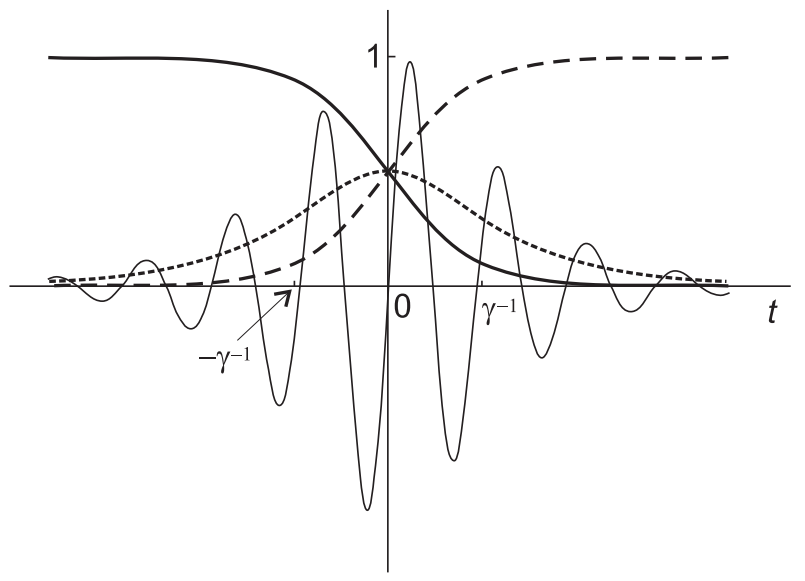

FIG. 2: Decay of quasi-stationary state: $\left|a_{1}\right|^{2}-$ thick solid curve, $\left|a_{0}\right|^{2}$ - dashed curve, $a$-dotted curve, and $x_{0}$ - thin solid curve.

induced during the quantum transition, and the thin solid line shows the classical coordinate of first ball in the chain $x_{0}(t)$ which is calculated by means of expression (19). As a matter of fact, this curve represents the coordinate of any ball in the chain because according to Eq. (6) the coordinate of the $n$-th ball can be expressed as

$$
x_{n}(t)=x_{0}(t-a n / v) .
$$

Therefore, during the decay of the quantum state a string excitation of the shape shown by thin solid line is emitted and travels away from the quantum oscillator with a constant velocity $v$. It can be interpreted as the classical analog of an emitted phonon. Naturally, the total energy carried by the string excitation is equal to the energy difference between the two quantum levels. Consequently, we may conclude that the quantum subsystem provides the energy quantization in the classical one.

It is worth mentioning that taking into account the norm conservation $\left(\left|a_{0}\right|^{2}+\left|a_{1}\right|^{2}=1\right)$ Eqs. (20) can be transformed into another set of equations governing the behavior of three real variables: the inversion $I=\left|a_{1}\right|^{2}-$ $\left|a_{0}\right|^{2}$ and the (complex) polarization $a$. The linearized version of these equations in the vicinity of the stationary state $n=0(I=-1)$ reads

$$
\dot{I}=-2 \gamma I, \quad \dot{a}=-\gamma a .
$$

It is remarkable that these equations coincide with those considered in the density matrix formalism [17], moreover, from our Eq. (27) we are able to extract the relaxation times: the longitudinal relaxation time is $T_{1}=1 / 2 \gamma$ and the perpendicular one is $T_{2}=1 / \gamma$. We see that these relaxation times obey the relation $T_{2}=2 T_{1}$ inherent to the pure state when no ensemble average is carried out and this fact confirms the adequacy of our attempt to describe a dissipative quantum system in the Schrödingerequation formalism. 
Two more comments are in order. First, according to Eqs. (22) we used the boundary condition at $t=-\infty$. This is related to the classical description of the chain excitations when there is no spontaneous emission. When solving the equations numerically one has to add some initial vibration field fluctuation in order to force the quantum transition.

Another point is related to the Fourier transform of the coordinate of the first ball

$$
x_{0}(\omega)=\int_{-\infty}^{\infty} d \omega e^{\beta \omega t} x_{0}(t) \sim \frac{\pi / 2 \gamma}{\cosh (\eta \pi / 2 \gamma)}
$$

which gives us the shape of the emission line. We see that close to the resonant frequency, when $|\eta| \ll 1$, the line shape is Lorentzian coinciding with the result following from a simple calculation based on the Fermi's golden rule. However, further away from the resonance, the line shape deviates from the Lorentzian and features exponential tails.

\section{RESONANT POWER ABSORPTION}

The following problem is that of the resonant power absorbtion which we calculated solving the equation set (20) using two-level

$$
\begin{aligned}
\dot{a}_{0} & =\beta \frac{\eta}{2} a_{0}+\beta(F-\beta \gamma a) a_{1}, \\
\dot{a}_{1} & =\beta \frac{3 \eta}{2} a_{1}+\beta\left(F+\beta \gamma a^{*}\right) a_{0}, \\
a & =a_{0} a_{1}^{*} .
\end{aligned}
$$

and three-level

$$
\begin{aligned}
\dot{a}_{0}= & \wp(\eta / 2-\alpha) a_{0}+\beta(F-\beta \gamma a) a_{1}, \\
\dot{a}_{1}= & \beta(3 \eta / 2-5 \alpha) a_{1} \\
& +\beta\left(F+ß \gamma a^{*}\right) a_{0}+\sqrt{2} \beta(F-\beta \gamma a) a_{2}, \\
\dot{a}_{2}= & ß(5 \eta / 2-13 \alpha) a_{1}+\sqrt{2} \beta\left(F+ß \gamma a^{*}\right) a_{1}, \\
a= & a_{0} a_{1}^{*}+\sqrt{2} a_{1} a_{2}^{*} .
\end{aligned}
$$

approximations. Note that we excluded a nonlinear term from the equations of the two-level approximation because in this case it leads only to an inessential shift of the resonant frequency. The power absorption was calculated by averaging the instantaneous power over the period of the external force, that is,

$$
P=-2 F \operatorname{Im} a \text {. }
$$

The power absorption in the two-level approximation as a function of the deviation $\eta$ is shown in Fig. 3 for various force amplitudes. Note that Eqs. (29) and (30) depend linearly on their parameters, therefore, by using an appropriate scaling of the time we may reduce the number of parameters by one. Therefore, we choose to express the quantities $F, P, \eta$ and $\alpha$ in $\gamma$ units.
We observe the following typical behavior: For a weak force $\left(F_{0}<0.5 \gamma\right)$ the shape of the resonance resembles a Lorentzian, and its peak value is increasing with increasing force. When the force exceeds the critical value $F_{0}=0.5 \gamma$ the peak stops growing and is flattened at the top due to the saturation at the critical power absorption value $P_{0}=0.5 \gamma$ indicated by the thin dotted line.

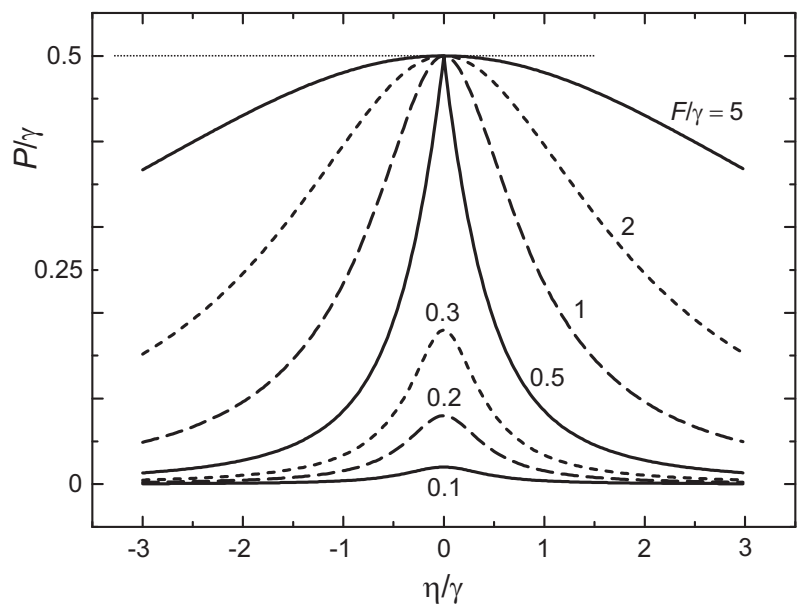

FIG. 3: Power absorption in the two-level approximation. The numbers on curves indicate the values of the external force amplitude $F / \gamma$.

In the next two Figures the results for power absorption calculated in the three-level approximation are shown. In Fig. 4 we plot the power absorption in a linear oscillator $(\alpha=0)$.

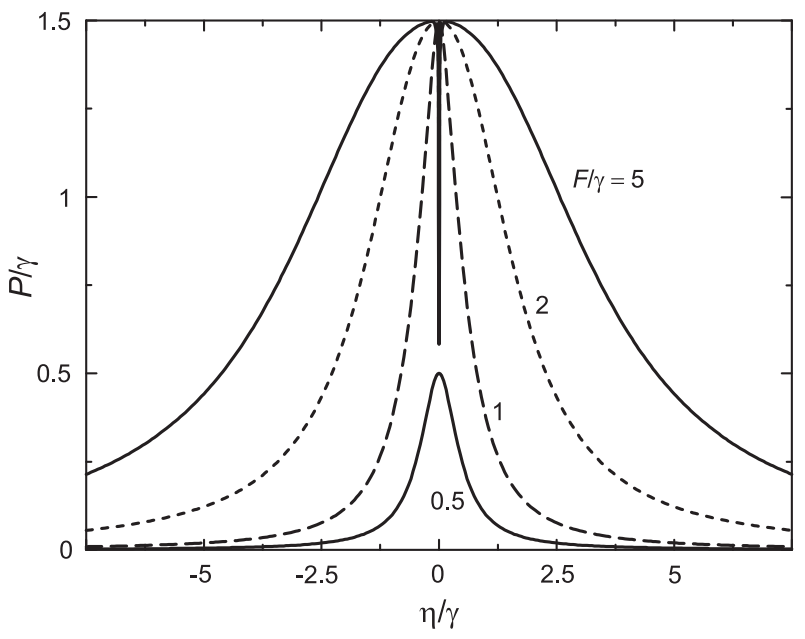

FIG. 4: Power absorption in the three-level approximation in the case of the linear oscillator. The numbers on the curves indicate the values of the external force amplitude $F / \gamma$.

The general shape of the absorption curves closely resembles those obtained in the two-level approximation, except that now the saturation sets in at a larger 
force value $F_{0}=\gamma$, and the saturated power absorption $P_{0}=1.5 \gamma$ is larger as well.

A more essential difference is seen in the uppermost curve corresponding to the force whose amplitude $(F=$ $5 \gamma$ ) strongly exceeds the critical value. Here we see a narrow gap in the absorption which appears close to the resonant frequency. It resembles an analogous gap that appears in the power absorption calculated by means of the density matrix equation technique [18] and is caused by the interaction of coherent light with an inhomogeneously broadened resonance line.

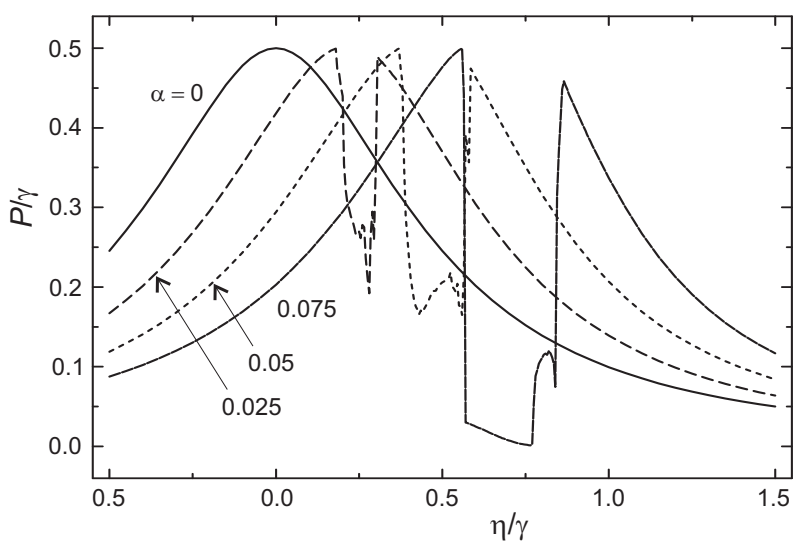

FIG. 5: Power absorption in the three-level approximation in the case of an anharmonic oscillator obtained for the parameter $F=0.5 \gamma$. The curves are numbered by the values of the anharmonicity coefficient $\alpha / \gamma$.

In Fig. 5 the results for the nonlinear oscillator are presented for a set of values of the anharmonicity coefficient and the force amplitude corresponding to the critical value of the two-level approximation, i. e. $F=0.5 \gamma$. We see that for the linear-oscillator case $(\alpha=0)$ the absorption demonstrates the expected Lorentzian behavior. When the anharmonicity is incremented the resonant peak moves to the right, becomes asymmetric and the saturation manifests itself as a widening absorption gap close to the shifted resonance frequency.

The absorption gap resembles the gap in nonlinear cyclotron resonance [19] which was described using the balance equations. In that work the gap was related to the dynamical chaos appearing in the classical nonlinear equations of motion. Quantum chaos is a subject of great interest as well (see [20] for references), and is largely concerned with the quantum signatures of classically chaotic systems. Such signatures are often sought by comparing the wave-function patterns corresponding to various eigenstates with the classical trajectories [21].

It follows from our quasiclassical consideration, Eqs. (20), that the behavior of the quantum system can also be visualized and interpreted in terms of phase-space trajectories obtained by treating the wave function expansion coefficients $a_{n}$ as generalized coordinates. In order to illustrate this possibility, we looked at the behavior of the quantum anharmonic oscillator in the absorption gap more carefully. In Fig. 6 the right-hand side of this absorption gap for the parameter values $F=0.5 \gamma$ and $\alpha=0.25 \gamma$ is shown in more detail. The simplest way to distinguish various types of dynamic behavior is to plot the phase portrait of the system. As there are three complex variables constrained by a single normalization condition the phase space is five-dimensional. Therefore, we

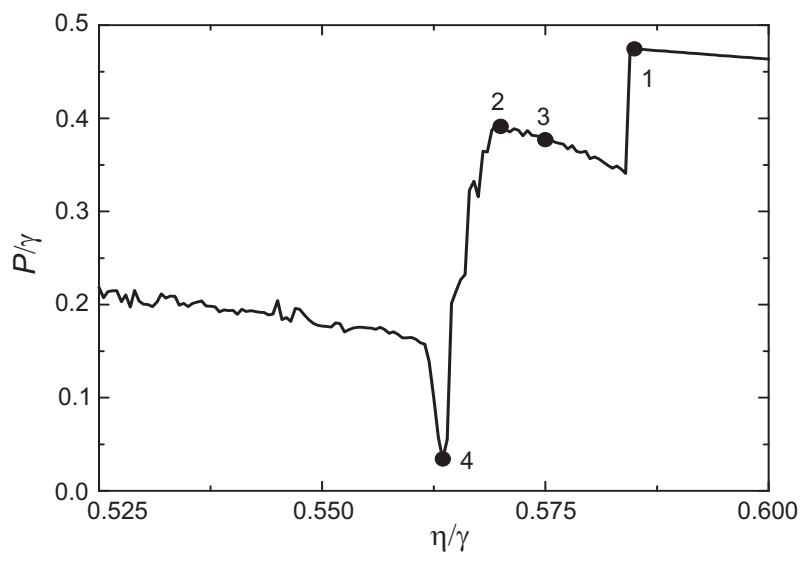

FIG. 6: Power absorption gap for the parameter values $F=$ $0.5 \gamma$ and $\alpha=0.25 \gamma$.

restrict ourselves to a projection of the phase space onto a two-dimensional plane $\left(\operatorname{Re} a_{0}, \operatorname{Re} a_{1}\right)$ shown in Fig. 7 . The panel numbers correspond to the frequencies indicated by dots in Fig. 6. We see that on the regular flank of
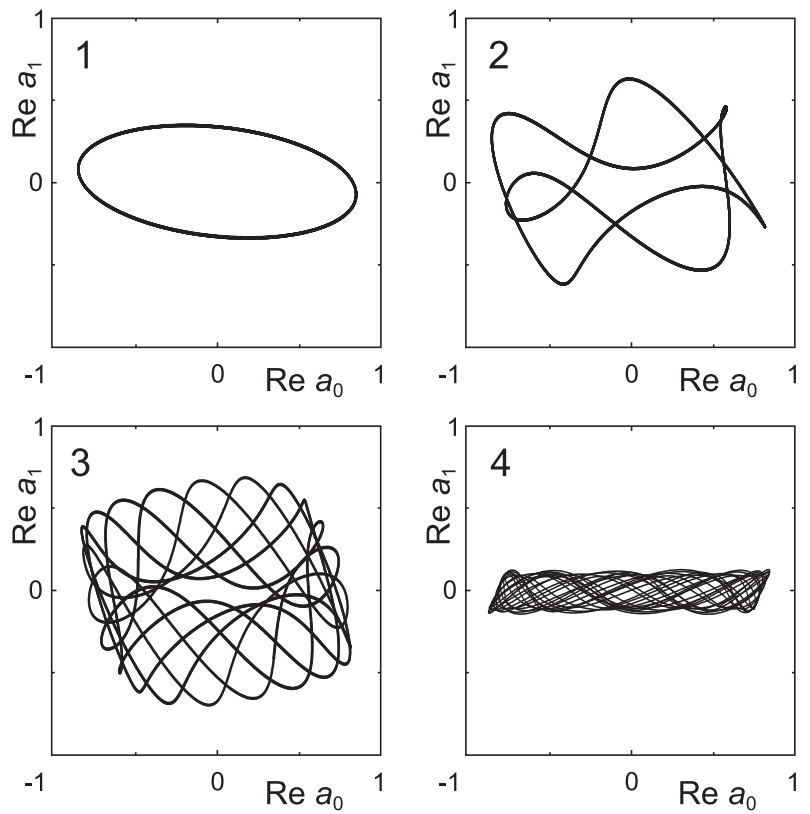

FIG. 7: Two-dimensional projection of the phase space. The numbers on the panels correspond to the frequencies indicated by dots in Fig. 6]

the resonance curve (point 1) the oscillator demonstrates 
a rather simple behavior - the limit cycle. As a matter of fact, in this case all three coefficients $a_{n}$ oscillate with the same frequency, namely, $a_{n}(t)=g_{n} \exp (B \zeta t)$. Therefore, in this case the power absorption problem can be simplified essentially and transformed into a nonlinear eigenvalue problem defining the frequency $\zeta$, and the stationary coefficients $g_{n}$ are simply related to the filling factors of the harmonic oscillator levels.

The behavior of the anharmonic oscillator in the absorption gap (points 2, 3,4) is quite different. Here, the coefficients $a_{n}$ oscillate with multiple frequencies, moreover, the oscillator can demonstrate both periodic behavior (point 2) and non-periodic behavior (points 3,4 ). This non-periodicity is in fact responsible for the gap in the power absorption as it causes the loss of coherence between the driving force and the system response. This is the so-called quasiperiodic behavior but the appearance of a strange attractor is also expectable at larger values of the anharmonicity coefficient $\alpha$.

\section{CONCLUSIONS}

In conclusion, we propose a Schrödinger-like equation for the description of a dissipative quantum-mechanical system. This equation is derived on the basis of a quasiclassical treatment of coupled quantum and classical subsystems and justified by the difference of masses of the two subsystems which leads to the smallness of adiabatic parameters. The considered decay of a quasistationary state demonstrates that the proposed description gives identical results to those obtained from the density-matrix approach when only homogeneous broadening is taken into account. The model calculation of the nonlinear absorption indicates that many classical phenomena - such as the asymmetry of the resonance shape and absorption dips - brought about by the loss of coherence between the driving force and the response may be featured by a quantum system as well. We believe that the proposed Schrödinger-equation formalism with dissipation may find application to quantum nanostructures and quantum chaos.

\section{Acknowledgments}

We thank Prof. K. Pyragas and J. Ruseckas for helpful discussions. This work was supported by the Lithuanian Science and Studies Foundation under grant No. T$05 / 18$.
[1] R. P. Feynman and F. L. Vernon Jr., Ann. Phys. 24, 118 (1963).

[2] A. O. Caldeira and A. J. Leggett, Ann. Phys. 149, 374 (1983).

[3] U. Weiss, Quantum Dissipative Systems, Series in Modern Condensed Matter Physics, vol. 10, (World Scientific, Singapore, 2006).

[4] F. Grossmann, J. Chem. Phys. 103, 3696 (1995), and references therein.

[5] S. M. Barnett and J. D. Cresser, Phys. Rev. A 72, 022107 (2005).

[6] S. Gao, Phys. Rev. Lett. 79, 3101 (1997).

[7] H. Dekker, Phys. Rev. A 16, 2126 (1977).

[8] D. Bohm, B. J. Hiley, and P. N. Kaloyerou, Phys. Rep. 144, 321 (1987).

[9] T. P. Spiller, P. S. Spencer, T. D. Clark, J. F. Ralph, H. Prace, R. J. Prance, and A. Clippingdale, Foundations of Physics Letters 4, 507 (1991).

[10] M. D. Kostin, J. Chem. Phys. 57, 3589 (1972).

[11] K. Albrecht, Phys. Lett. B 56, 127 (1975).
[12] R. Kapral and G. Ciccotti, J. Chem. Phys. 110, 8919 (1999).

[13] C.-C. Wan and J. Schofield, J. Chem. Phys. 116, 494 (2002).

[14] S. A. Egorov, E. Rabani, and B. J. Berne, J. Phys. Chem. B 103, 10978 (1999).

[15] A. Matulis and E. Anisimovas, Proceedings of International Workshop on Advanced Spectroscopy and Optical Materials, Gdansk, Poland, 2006 (to be published as a special issue of Optical Materials).

[16] W. Boucher and J. Traschen, Phys. Rev. D 37, 3522 (1988).

[17] K. Wódkiewicz, Phys. Rev. A 19, 1686 (1979).

[18] L. Allen and J. H. Eberley, Optical Resonance and TwoLevel Atoms, (Wiley, New York, 1975), Chap. 6.

[19] K. Pyragas, Sov. Phys. JETP 61, 1335 (1985).

[20] M. C. Gutzwiller, Am. J. Phys. 66, 304 (1998).

[21] E. J. Heller and S. Tomsovic, Physics Today 46 (7), 38 (1993). 